\title{
Einwirkungen des kalten Winters 1962/63 auf das Makrobenthos im Ostteil der Deutschen Bucht
}

\author{
ERICH Ziegelmeier \\ Biologische Anstalt Helgoland, Litoralstation, List/Sylt
}

\begin{abstract}
Effects of the cold winter 1962/63 on the macrobenthos in the eastern part of the German Bight. In the southern part of the North Sea, quantitative and qualitative samples of the macrobenthos fauna have been taken continuously since 1949. It was thus possible to assess the effects of the extremely low water temperatures during the cold winter 1962/63 (Fig. 1). Most benthonic species suffered considerable losses. Death rates were highest among the Lamellibranchiata, except for Macoma baltica and Nucula nitida (in both species mortality rates were less severe), and Echinodermata, especially Amphiura filiformis. Death rates were lower in Crustacea and lowest in Polychaeta. In general, death rates of benthonic animals appear to depend on water depth, type of substrate, affiliation to different zoogeographical fauna groups, body size and, presumably, also motility.
\end{abstract}

\section{EINLEITUNG}

Nach Beginn der qualitativen und quantitativen Bodenfaunauntersuchungen (Herbst 1949) im Ostteil der Deutschen Bucht wurde auf Grund der Ergebnisse in den ersten Jahren vermutet, daß der sehr strenge Winter 1946/47 das Makrobenthos in unserem Meeresgebiet stark dezimiert hatte (Zregremeier 1953). Obgleich keine Proben aus der Zeit vor 1949 zur Verfügung standen, ließ die Auswertung des Bodengreifermaterials aber deutlich erkennen, in welchem Maße die häufigsten Wirbellosen geschädigt wurden: Am stärksten die Mollusken (insbesondere die Lamellibranchiata) und Echinodermen, weniger die Crustaceen und Polychaeten. So setzte die allmähliche Wiederbesiedlung des Gebietes nach dem Winter 1946/47 mit einer sehr starken Anreicherung der vermutlich am meisten dezimierten Muschelarten ein. Dies führte im Herbst 1950 und Frühjahr 1951 zu Häufigkeitsmaxima besonders bei Aloidis gibba, Abra alba, A. nitida und Angulus fabula. Ampbiura filiformis, ein Schlangenstern, der nach CAspers (1938) in der Helgoländer Tiefen Rinne Bestandszahlen bis 144 Individuen in 0,1 $\mathrm{m}^{2}$ aufwies, war 1949 noch völlig verschwunden. Erst im Frühjahr 1955 konnte A. filiformis auf einem schlickigen Fangplatz nordwestlich von Helgoland in 32-34 m Tiefe mit langsam und stetig steigender Abundanz gefunden werden.

Einen Hinweis auf die Temperaturempfindlichkeit bei Echinodermen erbrachten bereits die Ergebnisse der Untersuchungen nach dem Winter 1955/56, dem kältesten in der Zeit von 1949 bis 1962 in unserem Gebiet (Zregelmerer 1963). Das Junggut 
von Echinocardium cordatum wurde vernichtet, bei den Mollusken, Crustaceen und Polychaeten waren nur geringe oder keine Ausfälle zu beobachten.

\section{ERGEBNISSE}

In dieser vorläufigen Mitteilung werden die Ergebnisse der 1963 mit F.K. „Uthörn" durchgeführten Untersuchungsfahrten im Ostteil der Deutschen Bucht und der Exkursionen im Nordsylter Wattgebiet in aller Kürze referiert. Ein Vergleich der bei List und in unmittelbarer Nähe des Feuerschiffes P 8 in den Jahren 1955/56 und

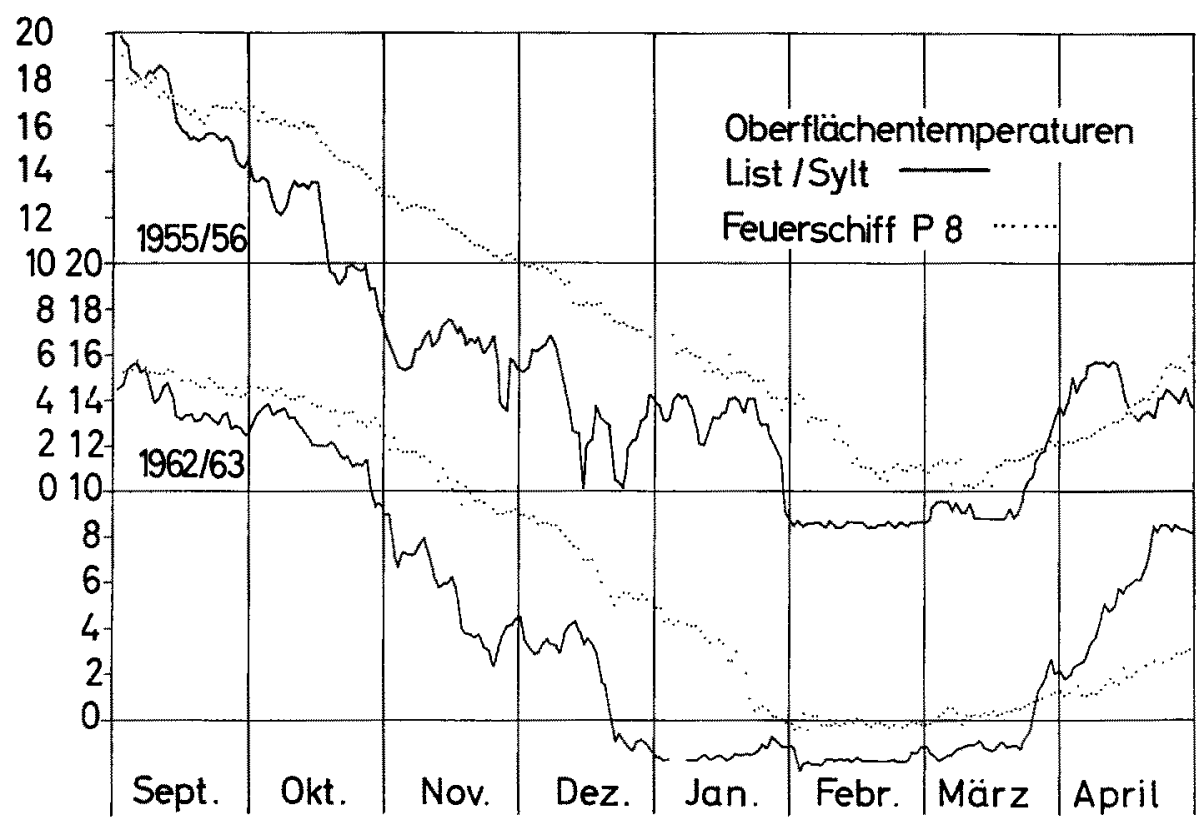

Abb. 1: Oberflächentemperaturen vom 1. September bis 30. April für 1955/56 und 1962/63. Mittelwerte von zwei täglich durchgeführten Messungen

1962/63 gemessenen Oberflächentemperaturen (Abb. 1) zeigt deutlich, daß die Temperaturen während des kalten Winters $1962 / 63$ erheblich niedriger waren. Schon dieser Umstand läßt eine entsprechend stärkere Schädigung der Makrobenthos-Fauna vermuten - eine Annahme, welche durch die Auswertung der Proben vollauf bestätigt wurde.

Auffallend war während der Untersuchungsfahrt in der Zeit vom 23. 4.-4. 5. 1963 auf den meisten Stationen bei den Probeentnahmen der mitunter starke Verwesungsgeruch der Baggerinhalte, den hauptsächlich die abgestorbenen Mollusken verursachten. Ein Maß für die Verluste bei den Weichtieren sind die frischen Doppelklappen der Muscheln und die frischen leeren Gehäuse der Schnecken im Siebrest der Bodengreiferfänge. Eine Zusammenstellung der ab 1955 ermittelten Durchschnittswerte von 


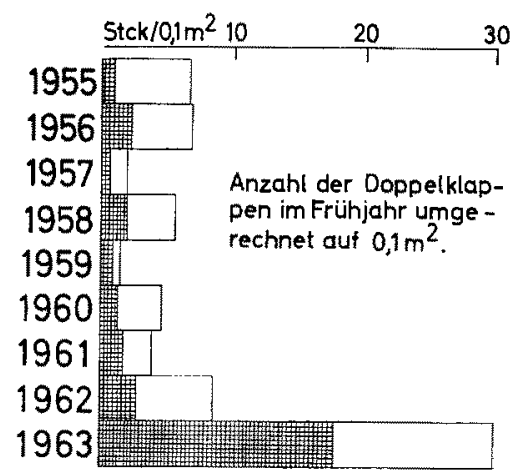

Angulus fabula

Abb. 2: Anzahl der Muscheldoppelklappen pro 0,1 $\mathrm{m}^{2}$ in den Jahren 1955 bis 1963 . Durchschnittswerte aus den Frühjahrsproben

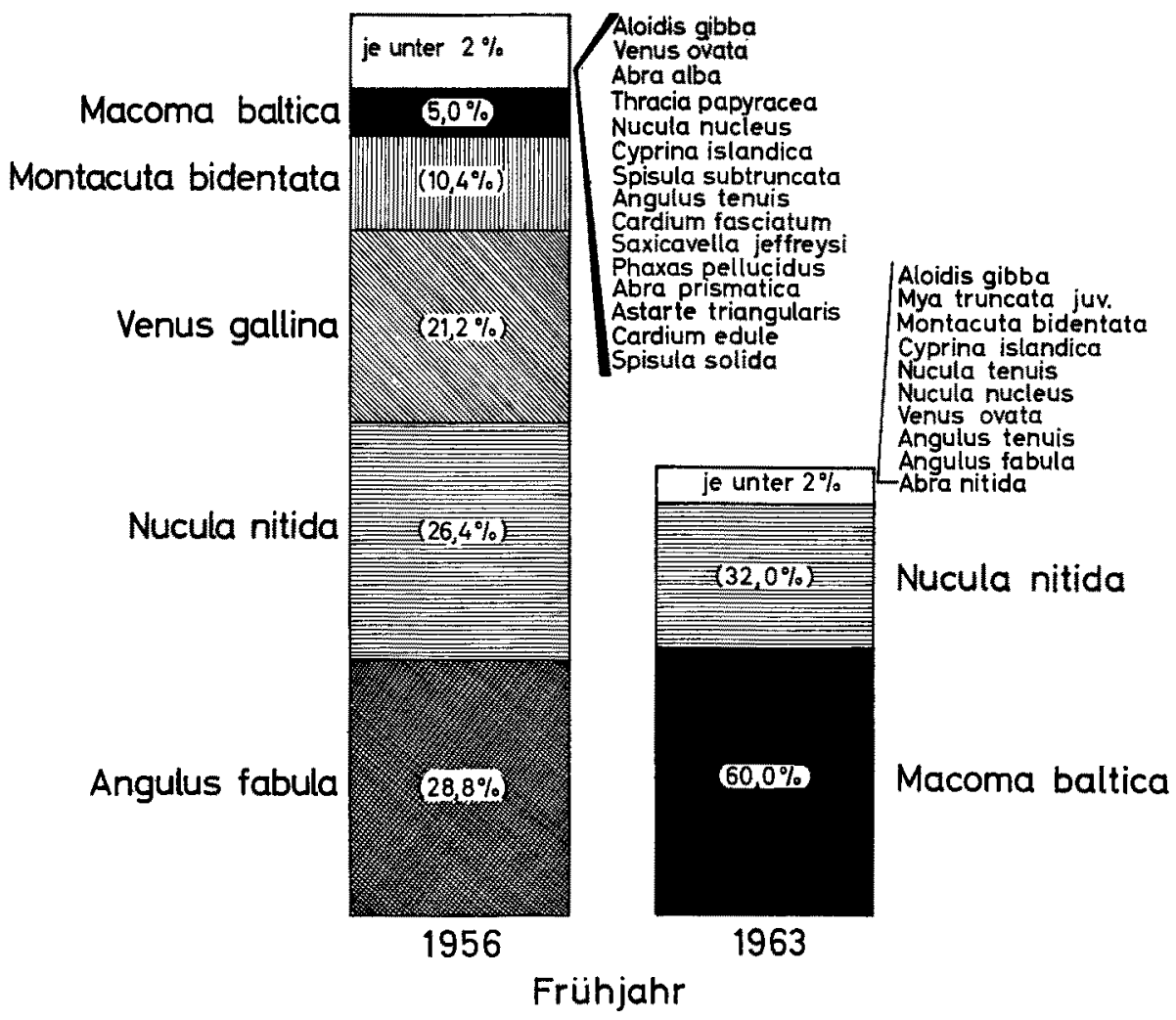

Abb. 3: Prozentuale Anteile der aus den Bodengreiferproben der Frïhjahrsfahrten 1956 und 1963 gesammelten, lebenden Lamellibranchiata-Arten 
Doppelklappen der Frühjahrsproben ergibt für 1963 etwa das 6fache an Schalen auf $0,1 \mathrm{~m}^{2}$ im Siebrest (Abb. 2). Mit mehr als 50\% ist Angulus fabula daran beteiligt, die in unserem Gebiet häufigste, konstant vorkommende und als Nahrungstier für Bodenfische wichtigste Muschelart (ZIEGELMEIER 1963). Abbildung 3 zeigt die prozentuale Verteilung aller aus den Bodengreiferproben der Frühjahrsfahrten 1956 und 1963 gesammelten lebenden Lamellibranchier-Spezies. Wie aus dieser vergleichenden Darstellung hervorgeht, wurde Angulus fabula fast völlig vernichtet. In den Baggerfängen vom Frühjahr 1963 konnte insgesamt nur eine einzige lebende $A$. fabula (Schalenlänge: $5,5 \mathrm{~mm}$ ) gefunden werden.

Bei der zu derselben Gattung gehörenden Angulus tenuis, die in Küstennähe bei geringen Tiefen in gröberen, gut überströmten Sanden häufig vorkommt, in den Bodengreiferproben der tieferen Plätze aber nur eine untergeordnete Rolle spielt, waren ebenfalls sehr große Ausfälle festzustellen, wie die Exkursionen im Nordsylter Wattgebiet nach dem Verschwinden der Eisschollen ergaben. Bei Ostwind und günstigen Niedrigwasserständen lagen die $A$. tenwis-Doppelklappen in großen Mengen (einigermaßen gleichmäßig verteilt) auf dem flachen Wattboden. Nach Stichproben an verschiedenen Stellen des Königshafenwatts bei List/Sylt betrug die Anzahl der frischen A. tenuis-Schalen 15 bis 40 Stück pro $\mathrm{m}^{2}$. Die Spülsäume am Wattstrand bestanden mitunter fast ausschließlich aus $A$. tenuis-Doppelklappen, weniger aus Cardium edule.

Nach den Bodengreiferproben vom Frühjahr 1963 haben nur 2 LamellibranchierArten den Eiswinter ohne oder doch nur mit geringen Schädigungen überstanden: Macoma baltica und Nucula nitida. Von Macoma baltica, der ausgesprochen borealen Küstenform, wurden in den Siebresten der Baggerproben nur ganz selten frische Doppelklappen gefunden. Die Bestände waren dichter, und auf einer Station in $10 \mathrm{~m}$ Tiefe betrug der Abundanzwert sogar etwa das 3fache von dem der Frühjahrsprobe 1962. Macoma konnte im Frühjahr 1963 auch im zähen Schlick in Tiefen bis $35 \mathrm{~m}$ festgestellt werden, in denen sie nach den Untersuchungen seit 1949 nie auftrat.

Nucula nitida, eine typische Schlickbewohnerin, die, mit einem kräftigen Grabfuß ausgestattet, im Substrat gut bewegungsfähig ist, wurde nur auf flachen Plätzen in größerer Anzahl tot aufgefunden. Im tieferen Wasser konnten bis 90 lebende $N$. nitida in einem $0,1 \mathrm{~m}^{2}$ Bodengreiferfang gezählt werden.

Von den prosobranchen Gastropoden ist in den Bodengreiferfängen Lunatia nitida die häufigste Art. Ihre Abundanz- und Praesenzwerte stiegen seit 1958, nach der Anreicherung der Muschelbestände, stetig an. Nach den mitunter zahlreichen frischen Leergehäusen im Siebrest der Proben vom Frühjahr 1963 und von einigen Materialbeschaffungsfahrten mit F.K. „Uthörn" zu urteilen, bei denen keine lebende Lunatia gefunden wurde - auch nicht an den Örtlichkeiten, die vorher reichlich besetzt waren -, ist diese Nabelschneckenart im letzten Winter sehr stark dezimiert worden. Während bei den Untersuchungsfahrten vor 1963 jeweils insgesamt bis zu 40 Lunatia aus den quantitativen Fängen gesammelt werden konnten, enthielten die Frühjahrsproben 1963 keine einzige lebende Nabelschnecke. Bei Litorina litorea im Watt und Buccinum undatum im tieferen Wasser waren keine Schädigungen zu beobachten.

Erhebliche Ausfälle konnten bei Echinodermen festgestellt werden, besonders bei den zur Infauna gehörenden Arten. Amphiura filiformis ist von den Fangplätzen bei Helgoland völlig verschwunden, auch im sandigen Schlick der Ampbiura-reichsten 
Station "Nordhafen innen", bei der in einer Baggerprobe $\left(0,1 \mathrm{~m}^{2}\right)$ vom Herbst 1962 noch 357 Schlangensterne gezählt werden konnten, waren im Februar 1963 keine Reste zu finden. Die meisten jungen Tiere der epibiotisch lebenden Ophizra-Arten O. texturata und $O$. albida waren am Leben geblieben, die größeren Exemplare aber sämtlich abgestorben. Von Ecbinocardium cordatum lagen entweder nur Bruchstücke verendeter Individuen oder zahlreiche im Sand verstreute Stacheln toter Tiere vor, und von Echinocyamus pusillus, dem kleinen Schildigel, fanden sich nur leere Gehäuse in den Bodengreiferproben. Während Asterias rubens durch die niedrigen Temperaturen kaum dezimiert wurde, fehlten in den Beifängen der Kuttertrawls im Frühahr 1963 gänzlich die im Sand eingegraben lebenden Kammsterne Astropecten irregularis.

Den Hauptanteil der Crustacea in den Bodengreiferproben bilden die Amphipoda. Die häufigsten Arten sind: Batbyporeia guilliamsoniana, Ampelisca brevicornis, Urothoe poseidonis, die im Sediment leben und häufig mit hoher Abundanz die sandigen und schlick-sandigen Böden besiedeln, ferner Nototropis swammerdami, zur Epifauna gehörend. Während von den ersten beiden Spezies im Frähjahr 1963 kein Exemplar gefunden wurde, trat Urotboe vereinzelt in den Proben auf. Neben einigen Funden von Nototropis in den Baggerproben, konnte bei einigen Kuttertrawlzügen im Frühjahr 1963 in den in Netzmaschen hängengebliebenen Hydroiden-Büscheln massenhaft $N$. swammerdami beobachtet werden. Von Cumaceen waren bei Diastylis rathkei nur sehr geringe Bestandswerte zu ermitteln. Alle anderen Crustaceen, wie Callianassa, Upogebia, Crangon allmanni und andere fehlten in den Bodengreiferfängen völlig.

Mit Ausnahme von Lanice conchilega und den Serpuliden sind die meisten Polychaetenarten durch die niedrigen Temperaturen am wenigsten geschädigt worden. Sämtliche vagilen Formen, wie Nephtbys, Scoloplos, Phyllodoce, Goniada, Ophelia und andere blieben am Leben, ebenso die kleinen tubicolen Arten Spiophanes und Myriochele; junge Owenia, Pectinaria koreni, P. auricoma, ferner Magelona. Von Lanice wurden aus den Bodengreiferproben viele Weichkörperteile, die vor der Fixierung bereits abgestorben waren, gefunden. Es handelte sich meist um Reste großer Tiere mit Wohnröhrendurchmesser um $6 \mathrm{~mm}$. Im Watt sind nach zahlreichen Kontrollen im März 1963 sämtliche adulte Lanice vernichtet worden. Erst Ende Juni 1963 wurden wieder junge lebende Lanice im Watt angetroffen mit Röhrendurchmessern von 2 bis $3 \mathrm{~mm}$. Es ist anzunehmen, daß diese Tiere, im Frühjahr 1962 geboren, als Juvenile ebenso den kalten Winter im Wattboden überstanden haben wie die kleinen Polychaetenarten Pygospio, Polydora, Heteromastus und infolge der verringerten Nahrungskonkurrenz schnell heranwuchsen, ähnlich wie der Quappwurm Echiurus echiurus, bei dem im Sommer 1963 im Ostteil der Deutschen Bucht und im Watt Massenvorkommen festgestellt werden konnten, die vorher nie zu beobachten waren. Bei Echiurus spielt offenbar auch die Reduktion der Muschelbestände (geringere Vernichtungsrate der Echiurus-Larven) eine Rolle.

\section{ZUSAMMENFASSUNG}

1. Am stärksten geschädigt wurden die zur Infauna zählenden Wirbellosen, wie die meisten Lamellibranchier, der Gastropode Lunatia, die Echinodermen Amphiura filiformis, Echinocardium cordatum, Astropecten irregularis, die Crustaceen Bathy- 

nachweisbar waren. Herr WeStherde führte im Rahmen seiner Arbeiten über die PolychaetenFamilie der Hesionidae vom 22. bis 25. März 1963 Untersuchungen am West- und Ostrand von List (Sylt) durch, welche mit entsprechenden Untersuchungen vom Oktober 1962 verglichen werden können. Bei Außentemperaturen von $\mathrm{O}^{0} \mathrm{C}$, einer Temperatur des freien Wassers von etwa $1^{\circ} \mathrm{C}$ und des Küstengrundwassers yon $1,5^{\circ} \mathrm{C}$ war im genannten Zeitraum des Monats März die Eisdecke überall aufgerissen, so daß Sandproben in beliebiger Entfernung von der Niedrigwasserlinie entnommen werden konnten. Zwar wurden einige Sandpolychaeten nicht gefunden (womit ihre Existenz zu dieser Jahreszeit noch keineswegs auszuschließen ist) und die Archianneliden (Protodrilus, Trilobodrilus) waren nur spärlich vertreten. Unter den Polychaeten traten jedoch Jungtiere von Nereis diversicolor und die Art Ophelia cluthensis (juvenil und adult), unter den Turbellarien z. B. die Proseriata Nematoplana coelogynoporoides und Notocaryoplanella glandulosa mit ungefähr gleicher Abundanz wie im Herbst 1962 auf. Neben verschiedenen Ostracoden und Harpacticiden wurden ferner Ciliaten, Nematoden und Oligochaeten ohne merkliche Veränderung ihrer Individuenzahl gefunden. Insgesamt ergaben sich somit gegenüber dem Oktober 1962 keine auffälligen Abweichungen in der Zusammensetzung der Mikrofauna. Herr Dörjes arbeitete vom 1. April bis 6. Mai und vom 1. August bis 7. September 1963 auf Helgoland über die Turbellarien der Ordnung Acoela. In sublitoralen Lebensräumen der unmittelbaren Umgebung Helgolands $(5-10 \mathrm{~m}$ Tiefe) lag der Artbestand im April mit der bemerkenswerten Zahl von 40 (!) nur wenig niedriger als im September (47 Arten), wobei auch die quantitativen Ausbeuten gut vergleichbar waren. Für einzelne tiefer gelegene Regionen überstiegen die Artenzahlen im Frühjahr die entsprechenden Verhältnisse im Herbst sogar ganz beträchtlich. Ich nenne für den AmphioxusSand (20 m Tiefe) 6 Arten im April, 2 im September; für den Nordhafen (bis $35 \mathrm{~m}$ Tiefe) die entsprechenden Werte 6 und 3; für die Tiefe Rinne (bis $60 \mathrm{~m}$ Tiefe) sagar 12 Arten im Frühjahr gegenüber nur einer Art im September. Eine Erklärung für diese auffällige Häufung von sublitoralen Turbellaria Acoela im Frühjahr können wir bis zur näheren Kenntnis der Lebenszyklen und der geographischen Verbreitung - es handelt sich vorwiegend um neue, noch unbeschriebene Arten - nicht geben.

GeRLACH: Auch ich habe den Eindruck gewonnen, daß der kalte Winter unter der Mikrofauna des Wattenmeeres und der Kieler Bucht keine nennenswerten Schäden verursacht hat. Am 25. Februar 1963 untersuchte idh gemeinsam mit Herrn Dr. Murphy das Sandwatt vor Büsum und das Schlickwatt bei Warwerort. Soweit man sehen konnte, waren die Watten von einer fast glatten, etwa $50 \mathrm{~cm}$ dicken Eisdecke überzogen, ohne daß nennenswerte Spalten auftraten. Nach dem Durchschlagen der Eisdecke wurde beobachtet, daß (einige Stunden nach Niedrigwasser) Wasser schnell von unten her das Loch füllte, so daß wahrscheinlich zwischen Eisdecke und Wattboden bei Hochwasser eine Wasserzirkulation stattfindet. Proben vom Wattensediment enthielten lebende Nematoden, Harpacticiden und Ostacoden in Individuenzahlen, wie sie etwa auch im Sommer auftreten, dazu Turbellarien, Oligochaeten und Pygospio elegans. - Am 28. März 1963, wenige Tage, nachdem das Eis der Kieler Förde aufgebrochen war, konnten wir am Strand bei Schilksee zahlreiche lebende Nematoden und andere Vertreter der Mikrofauna im Feinsand bei $50 \mathrm{~cm}$ Wassertiefe finden. Ebenso erwiesen sich am 8 . April 1963 mit dem Forschungskutter "Hermann Wattenberg" in der sublitoralen Region der Kieler Bucht gesammelte Proben in sandigen und schlickigen Sedimenten als reich besiedelt. - Im Gegensatz hierzu stehen Beobaditungen vom 3. April 1963 aus Helgoland. Proben aus der sublitoralen Region östlich und südlich der Düne (Amphioxus-Sand und andere Sedimente) waren nur spärlich besiedelt. Allerdings fehlen uns hier bislang vergleichende Untersudhungen aus den Sommermonaten, und darüber hinaus muß offenbleiben, ob gegebenenfalls die Veränderung des Lebensraums durch von abschmelzenden Eisschollen stammende Sinkstoffe oder abgestorbene Muscheln einen Einfluß auf die Mikrofauna hatte.

ZIEGfLMEIER: Wir haben Vergleichsmöglichkeiten, allerdings keinen Bearbeiter. Es stehen quasi-quantitative Meiobenthos-Proben aus der Zeit von Herbst 1949 bis heute in List zur Verfügung. Wenn sie einmal ausgewertet sind, werden auch diese Fragen, hoffe ich, beantwortet werden können. 